\title{
Trefftz-type approach for 2D fracture problems
}

\author{
A. N. Galybin \\ Wessex Institute of Technology, Southampton, UK
}

\begin{abstract}
This study is aimed at the development of a Trefftz-type method for solving plane elastic boundary value problems for open contours, which models crack propagation in brittle materials. The idea of the approach is as follows. Complex potentials are sought as linear combinations of independent holomorphic functions with the weights addressing singularities at the crack tips. Then the collocation method is applied to satisfy boundary conditions, which leads to a linear system for determination of unknown coefficients in the representation for complex potentials. The system is, in general, overdetermined and, thus, the SVD regularisation is applied to find its approximate solution. Two examples are presented.
\end{abstract}

Keywords: cracks, complex potentials, Trefftz method, collocations, ill-posed problems.

\section{Introduction}

Common technique for solving plane elastic problems with cracks assumes determination of two holomorphic functions (complex potentials, see Muskhelishvili [1]) that, in general, have square root singularities at crack tips. Usually, complex potentials are found by solving singular integral equation, SIE, (or a system of SIEs) with respect to unknown generalised crack opening displacements or their densities. This approach requires discretisation of the boundary and application of special quadratures for singular integrals, which represent two common steps in boundary integral methods. The present study is aimed to avoid these two steps and to apply directly the Trefftz approach by representing complex potentials as linear combinations of known holomorphic functions. 
The Trefftz approach for elastostatics is known and its different variants have been discussed [2- 6]. However application of the method for fracture mechanics problems (e.g., [4,5]) is not fully developed. Here the approach used in [6-7] for smooth contours and non-classical formulations is extended for the case of plane bodies with cracks. It is proposed to seek representations for complex potentials as linear combinations of independent holomorphic functions, that have square root singularities at the crack ends, followed by the determination of unknown (complex) coefficients from the boundary conditions by the collocation method. In general, the number of collocation points is assumed to be greater than the number of the sought coefficients, which results in an overdetermined system of linear algebraic equations. An approximate solution of the system is obtained by regularisation of the matrix by using the singular value decomposition method. The regularisation is important because it ensures that only linearly independent holomorphic functions are used as the basis functions in series for complex potentials. Two examples that use different sets of basis functions are presented to illustrate the approach. These are for a crack in a plane and acrack in a circle.

\section{Trefftz approach for cracks in 2D elastic media}

\subsection{General expressions for the Trefftz approach using complex potentials}

General solution (no body forces) for plane elastic domain has the form [1]

$$
\begin{aligned}
& \frac{\sigma_{11}+\sigma_{22}}{2}=P(z, \bar{z})=\Phi(z)+\overline{\Phi(z)} \\
& \frac{\sigma_{22}-\sigma_{11}}{2}+i \sigma_{12}=D(z, \bar{z})=\bar{z} \Phi^{\prime}(z)+\Psi(z) \\
& 2 G\left(u_{1}+i u_{2}\right)=W(z, \bar{z})=\kappa \varphi(z)-z \overline{\Phi(z)}-\overline{\psi(z)}, \Phi(z)=\varphi^{\prime}(z), \Psi(z)=\psi^{\prime}(z)
\end{aligned}
$$

Here $\varphi, \psi, \Phi$ and $\Psi$ are arbitrary holomorphic functions that are to be found from boundary conditions; harmonic function $P$ and complex-valued function $D$ represent mean stress and stress deviator which are functions of stress components $\sigma_{i j} ; W$ is a complex-valued function proportional to the displacement vector $\left(u_{1}, u_{2}\right) ; G$ is the shear modulus, $\kappa=3-4 v$ for plane strain and $\kappa=(3-v) /(1+v)$ for plain stress, $v$ is Poisson's ratio.

Boundary value problem for cracks in plane elastic bodies is formulated as the first boundary problem of elasticity for which tractions (or stress vector) is known on the crack. In terms of stress functions and complex potentials this problem can be presented in the following form [1]

$$
P(\zeta)+\frac{\overline{d \zeta}}{d \zeta} \overline{D(\zeta)}=2 \operatorname{Re}(\Phi(\zeta))+\frac{\overline{d \zeta}}{d \zeta}\left(\zeta \overline{\Phi^{\prime}(\zeta)}+\overline{\Psi(\zeta)}\right)=N(\zeta)+i T(\zeta), \quad \zeta \in \Gamma
$$

Here $N(\zeta)$ and $T(\zeta)$ are normal and shear components of stresses on the boundary $\Gamma$ of the considered domain $\Omega$ (interior or exterior including the crack); $P(\zeta)$ and $D(\zeta)$ are boundary values of the stress functions defined via boundary values of complex potentials $\Phi(\zeta)$ and $\Psi(\zeta)$. In fracture mechanics, in general, 
condition (2) is valid on the contour of the crack only, while on the rest of the boundary one can use mixed boundary conditions assuming that either tractions or displacements are known on different parts. Here, for the sake of simplicity, we apply (2) to the entire contour $\Gamma$.

Complex potentials $\Phi(z)$ and $\Psi(z)$ are sought in the form

$$
\varphi(z)=\sum_{k=1}^{n} a_{k} h_{k}(z), \quad \psi(z)=\sum_{k=1}^{n} b_{k} h_{k}(z)
$$

where $h_{k}(\mathrm{z})$ are independent functions holomorphic in the entire domain $\Omega ; 2 n$ complex coefficients $a_{k}$ and $b_{k}$ are unknown (for simplicity the number of terms in both sums are the same). To satisfy (2) one needs to find derivatives of (3); they can be presented in the form

$$
\Phi(z)=\sum_{k=1}^{n} a_{k} H_{k}(z), \quad \Phi^{\prime}(z)=\sum_{k=1}^{n} a_{k} H_{k}^{\prime}(z), \quad \Psi(z)=\sum_{k=1}^{n} b_{k} H_{k}(z)
$$

where $H_{k}(z)=h_{k}^{\prime}(z)$ are holomorphic in $\Omega$.

Substitution of (4) into conjugated boundary condition (2) yields the following expression

$$
\sum_{k=1}^{n}\left\{2 \operatorname{Re}\left(a_{k} H_{k}(\zeta)\right)+\frac{d \zeta}{\overline{d \zeta}}\left(a_{k} \bar{\zeta} H_{k}^{\prime}(\zeta)+b_{k} H_{k}(\zeta)\right)\right\}=N(\zeta)-i T(\zeta), \quad \zeta \in \Gamma
$$

Unknown coefficients $a_{k}$ and $b_{k}$ can be determined from a system of linear algebraic equations obtained from (5) by diverse methods, for instance by collocations, which is accepted in the Trefftz approach.

\subsection{Modification for cracks}

For the case when contour $\Gamma$ has sharp corners direct application of (5) is limited. This is explained by the presence of weak singularities in functions $H_{k}(\mathrm{z})$, which may lead to strong singularities in $H_{k}^{\prime}(\mathrm{z})$. In fact, in the case of cracks some of the functions $H_{k}(\mathrm{z})$ (or all of them) have square root singularities at the crack tips and the derivatives will have singularities of order $-3 / 2$. Therefore, a modification of (5) is necessary to avoid the presence of unphysical singularities, which can be done in the following way. Firstly, we introduce a special holomorphic function $\chi(z)$ that nullifies the difference $\bar{\zeta}-\chi(\zeta)$ at all crack tips and other sharp corners. Then the stress deviator (second formula in (1)) can be presented in the form

$$
D(z, \bar{z})=(\bar{z}-\chi(z)) \Phi^{\prime}(z)+\omega(z), \quad \omega(z)=\chi(z) \Phi^{\prime}(z)+\Psi(z)
$$

where $\omega(z)$ is a new unknown holomorphic function in $\Omega$. We further seek this function in the form

$$
\omega(z)=\sum_{k=1}^{n} a_{k+n} H_{k}(z)
$$


Thus, the function $\omega(z)$ (and consequently the stress deviator) has no strong singularities at the crack ends and sharp corners, and hence all stress components can only have singularities not stronger than square root.

The notation for unknown coefficients in (7) has been introduced for convenience and allows one to represent the modified expression (5) in the following form

$$
\begin{aligned}
\sum_{k=1}^{n}\left\{2 \operatorname{Re}\left(a_{k} H_{k}(\zeta)\right)+a_{k} \bar{\zeta} \frac{d \zeta}{d \zeta} H_{k}^{\prime}(\zeta)\right\}+ & \\
& +\sum_{k=n+1}^{2 n} a_{k} \frac{d \zeta}{\overline{d \zeta}} H_{k-n}(\zeta)=N(\zeta)-i T(\zeta), \quad \zeta \in \Gamma
\end{aligned}
$$

By introducing real coefficients

$$
a_{k}=C_{k}+i C_{2 n+k}, \quad k=1 \ldots 2 n
$$

one can rewrite (8) in the form

$$
\sum_{k=1}^{4 n} F_{k}(\zeta) C_{k}=p(\zeta), \quad \zeta \in \Gamma
$$

Here the following notation has been introduced

$$
F_{k}(\zeta)=\left\{\begin{array}{cc}
H_{k}(\zeta)+\overline{H_{k}(\zeta)}+\bar{\zeta} e^{2 i \theta(\zeta)} H_{k}^{\prime}(\zeta) & 1 \leq k \leq n \\
i\left(H_{k}(\zeta)-\overline{H_{k}(\zeta)}-\bar{\zeta} e^{2 i \theta(\zeta)} H_{k}^{\prime}(\zeta)\right) & n<k \leq 2 n \\
e^{2 i \theta(\zeta)} H_{k-n}(\zeta) & 2 n<k \leq 3 n \\
i e^{2 i \theta(\zeta)} H_{k-n}(\zeta) & 3 n<k \leq 4 n
\end{array}\right.
$$

is the set of known functions expressed via basis functions $H_{k}(\mathrm{z})$;

$$
p(\zeta)=N(\zeta)-i T(\zeta)
$$

is known stress vector on the contour;

$$
e^{2 i \theta(\zeta)}=\frac{d \zeta}{d \zeta}, \quad \zeta \in \Gamma
$$

where $\theta$ is the angle between the tangent to the contour at point $\zeta$ and real axis of a Cartesian coordinate system.

\subsection{On independence of coefficients $C_{k}$}

Unknown coefficients in (3) may be dependent. This is evident from Savruk's representation [8] of complex potentials via the Cauchy integrals

$$
\Phi(z)=\frac{1}{2 \pi} \int_{\Gamma} \frac{Q(t)}{t-z} d t, \quad \Psi(z)=\frac{1}{2 \pi} \int_{\Gamma}\left\{\frac{\overline{Q(t)}-2 i \overline{q(t)}}{t-z} \overline{d t}-\frac{\bar{t} Q(t)}{(t-z)^{2}} d t\right\}
$$

where $2 q(t)$ is a jump of $N+i T$ across the contour. If this jump vanishes on $\Gamma$ then both complex potentials are expressed via the density of the crack opening displacements $Q(t)=2 G(1+\kappa)^{-1}\left(u^{\prime+}{ }_{1}-u^{\prime-}{ }_{1}+i\left(u^{\prime+}{ }_{2}-u^{\prime-}{ }_{2}\right)\right)$ alone. This function can be 
sought as a finite series, which after integration results in the following expressions

$$
\begin{aligned}
& Q(t)=\sum_{k=1}^{n} a_{k} q_{k}(t), \quad \Phi(z)=\sum_{k=1}^{n} a_{k} H_{k}(z), \quad \Psi(z)=\sum_{k=1}^{n} \bar{a}_{k} G_{k}(z)-a_{k} T_{k}(z) \\
& H_{k}(z)=\frac{1}{2 \pi} \int_{\Gamma} \frac{q_{k}(t)}{t-z} d t, \quad G_{k}(z)=\frac{1}{2 \pi} \int_{\Gamma} \frac{q_{k}(t)}{t-z} \overline{d t}, \quad T_{k}(z)=\frac{1}{2 \pi} \int_{\Gamma} \frac{\bar{t} q_{k}(t)}{(t-z)^{2}} d t
\end{aligned}
$$

Here the coefficients $b_{k}$ are absent but different sets of basis functions is used for the representations of the second complex potential. The functions $G_{k}(\mathrm{z})$ and $T_{k}(\mathrm{z})$ can be expressed through $H_{k}(\mathrm{z})$ if $n$ tends to infinity, which will indicate dependence of the coefficients. If $n$ is finite then, in general, the coefficients can be considered as independent, at least with certain accuracy. However, the coefficients $b_{k}$ can be expressed through the coefficients $a_{k}$ in some cases of simple contours. This becomes evident if one considers a straight crack in the plane, in this case the solution can be derived via the potential $\Phi(z)$ only [1], which indicates that all coefficients $b_{k}$ in (3) depend on $a_{k}$. In complex cases of geometry this does not take place, however one cannot expect that all coefficients $C_{k}$ will be independent of each other for particular problems. Complete investigation of independence of representations (3) and/or (7) presents essential difficulties and it is out of scope of this paper. It is further accepted that in numerical calculations any dependence of the coefficients will manifests itself in ill-condition matrix of linear algebraic system. Therefore, it necessitates application of a regularisation technique regardless of the method used for solution of (10).

\subsection{Regularised approximate solution of the problem}

Method of collocation is further used to solve (10). For this purpose, $N$ nodes are selected on the boundary of the domain $\zeta_{j} \in \Gamma$, which results in the following linear algebraic system of equations

$$
\sum_{k=1}^{4 n} F_{k j} C_{k}=p_{j}, \quad j=1 \ldots N ; \quad F_{k j}=F_{k}\left(\zeta_{j}\right), \quad p=p\left(\zeta_{j}\right),
$$

The coefficients of this system and the right hand side are both complex, which is not suitable for the SVD method used to solve (16). Therefore, the following system has been formed

$$
\mathrm{MC}=\mathbf{B}
$$

where $\mathbf{C}$ is $4 n$-vector of unknowns with the components $C_{k}, \mathbf{M}$ is $2 N x 4 n$ matrix of the system, $M_{k m}=\operatorname{Re}\left(F_{k j}\right), m=1 \ldots N, M_{k m}=\operatorname{Re}\left(F_{k j}\right) ; m=N+1 \ldots 2 N ., k=1 \ldots 4 n ; \mathbf{B}$ is $2 N$-vector of applied load with the components $B_{m}=\operatorname{Re}\left(p_{m}\right), m=1 \ldots N ; B_{m}=\operatorname{Im}\left(p_{m}\right)$, $m=N+1 \ldots 2 N$.

The number of collocations is chosen to make system (17) overdetermined. Then the inverse matrix $\mathbf{M}^{-1}$ in (17) is found by the singular value decomposition, SVD (see e.g. Golub and van Loan [9]). This method allows to 
control the condition number of the matrix $\operatorname{Cond}(\mathbf{M})$ and if necessary to perform regularisation if $\operatorname{Cond}(\mathbf{M})$ is greater than a chosen threshold, $C^{*}$. The solution of (17) is presented as follows

$$
\mathbf{C}=\mathbf{M}_{r e g}^{-1} \mathbf{B}, \quad \mathbf{M}_{r e g}^{-1}=\mathbf{V}^{T} \mathbf{D}^{\prime} \mathbf{U}
$$

Here $\mathbf{U}(4 n \times 2 N)$ and $\mathbf{V}(4 n \times 4 n)$ are orthogonal matrices in the SVD of the matrix $\mathbf{M}, \mathbf{M}=\mathbf{U D V}^{T}, \mathbf{D}$ is $(4 n \times 4 n)$ diagonal matrix formed from the singular values, $d_{j}$, of the matrix $\mathbf{M}$ placed in descending order, $d_{1} \geq d_{2} \geq \ldots \geq d_{n}, \quad \mathbf{D}^{\prime}$ is the diagonal matrix of the rank $k$ as follows $\mathbf{D}^{\prime}=\operatorname{diag}\left\{d_{1}{ }^{-1}, d_{2}{ }^{-1}, \ldots d_{k}{ }^{-1}, 0 \ldots 0\right\}$. It should be noted that $\mathbf{D}^{\prime}=\mathbf{D}^{-1}$ if no regularisation has been made.

\section{Numerical examples}

\subsection{Crack in a plane}

Let us consider an elastic isotropic plane with a crack lying on the interval $(-L, L)$ of the real axis. Let the surfaces of the crack be subjected to normal and shear $\operatorname{load} p=N+i T$ (Fig 1a). An analytical solution for this configuration can be found, in particular, stress intensity factors, SIFs, at the right crack tip are found in the following form [8]

$$
K_{I}^{i d e a l}-i K_{I I}^{\text {ideal }}=\frac{-1}{\sqrt{\pi L}} \int_{-L}^{L} \sqrt{\frac{L+t}{L-t}} p(t) d t
$$

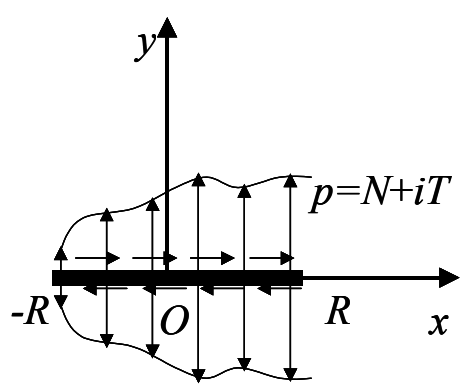

(a)

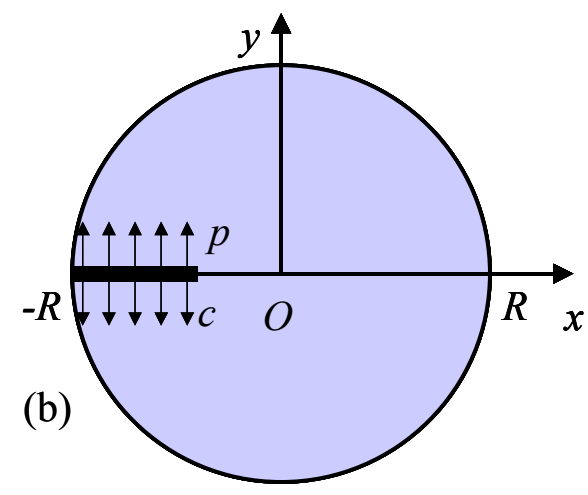

Figure 1: $\quad$ Crack in the plane (a), edge crack in the circle (b).

The following basis functions are used

$$
H_{k}(z)=k\left(\sqrt{z^{2}-L^{2}}-z\right)^{k-1}\left(\frac{1}{\sqrt{z^{2}-L^{2}}}-1\right)
$$

These provide correct behaviour of sought solutions at infinity and at the crack tips, which is important because improper choice of basic functions may lead to incorrect solutions. 
Calculated stress intensity factors are found in the form

$$
\frac{K_{I}-i K_{I I}}{p_{0} \sqrt{\pi L}}=\sum_{k=1}^{n}(-1)^{k-1} k\left(2 \operatorname{Re}\left(a_{k}\right)+a_{n+k}\right) L^{k-1}
$$

where $p_{0}$ is a parameter of loads.

Calculations have been performed for different polynomial loads, $p(t)=\alpha_{m}(x / L)^{m}$ with different $n$ and $N$. The condition number has been kept below $10^{7}$. The results for SIFs are accurate if $m<n$, which is explained by proper choice of basis functions (in this case $p_{0}=\alpha_{m}$ ). Tests have been also made for other load types and they show good agreement with analytical solutions. Thus solutions for step-like load on the crack have been checked. Fig 2 shown one example in which $n=36, N=576, C^{*}=212$ and calculates SIF has an error of less than $0.8 \%, K_{\mathrm{I}}^{\text {ideal }}=1 / 3(p L)^{1 / 2}$.

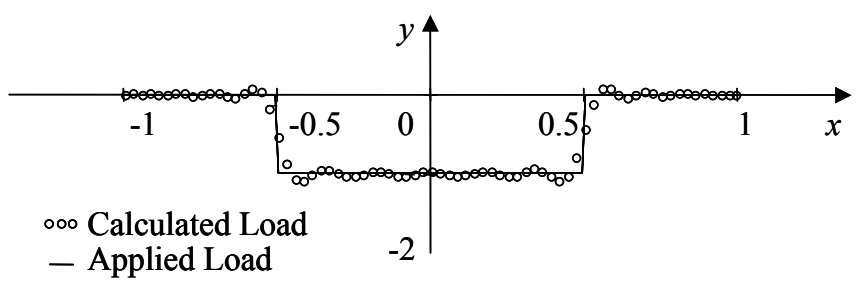

Figure 2: $\quad$ Boundary stresses for the case for step-like load applied to the crack in plane.

\subsection{Edge crack in a circle}

Let us consider a crack (of the length $L$ ) in an elastic circle of the radius $R$; the crack is located on the interval $(-R,-R+L)$ of the real axis, Fig $2 \mathrm{~b}$. The surfaces of the crack are subjected to normal pressure $p$. An analytical solution for this configuration can be found, in particular, mode I stress intensity factor is found in the following form [8]

$$
K_{I}^{i d e a l}=3.17214(2-L)^{-\frac{3}{2}} p \sqrt{\pi L}
$$

The following basis functions are used in modelling

$$
H_{k}(z)=(z-c)^{\frac{k}{2}-1}
$$

This selection provides correct behaviour of the solution at the crack tip and at the crack mouth.

Calculated mode I SIF is found as follows

$$
K_{I}=\left(\frac{a_{1}}{2}+\bar{a}_{1}+a_{n+1}\right) \sqrt{2} \pi p
$$

Calculations have been performed for different crack lengths varying from $0.2 R$ to $1.8 R$. Different combination of parameters $n$ and $N$ have been checked and in all tests the threshold for the condition number has been set $C^{*}=10^{8}$. The 
results for $K_{\mathrm{I}}$ are satisfactory for $0.2<L / R<1.4$ and the errors can be made within $1 \%$ by the proper selection of parameters $n$ and $N$. For long cracks the numbers $n$ and $N$ should increase, which makes the condition number of the matrix to be quite big and therefore the SVD regularisation cannot produce accurate results. An example of boundary stress calculations is presented in Fig 3 for the case $L=1.2 R$, where for $n=36, N=468$ (180 nodes have been selected on the circumference and $4 n$ nodes on the upper and lower boundaries of the crack, i.e. 288 nodes in total the contour modelling the crack). The condition number of the matrix $\mathbf{M}$ is greater than $10^{15}$, therefore the regularisation has been performed, Cond $\left(\mathrm{M}_{\mathrm{reg}}{ }^{-1}\right)=6.85 \cdot 10^{7}$. Despite reduced accuracy caused by regularisation the result for $K_{\mathrm{I}}=4.437(\pi L)^{1 / 2} p$ is within $0.1 \%$ error.
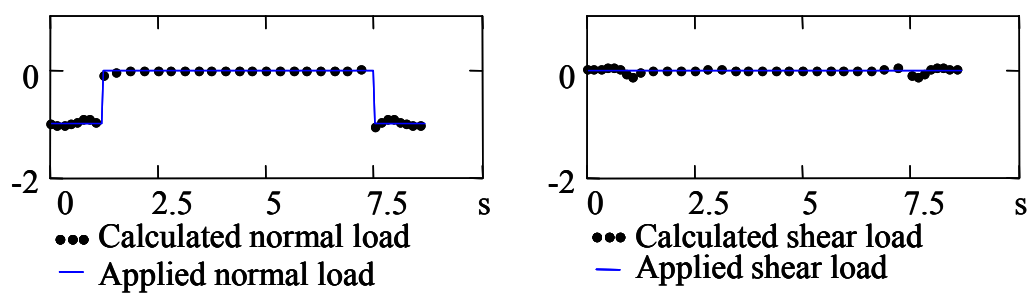

Figure 3: Normal (lefts) and shear (right) stresses on the contour in the case of the edge crack in the circle, $L=1.2 R$.

\section{Conclusions}

It is shown that the Trefftz approach in which complex potentials are approximated by linear combinations of holomorphic functions can be used for solving fracture mechanics problems. It is important to ensure correct behaviour of the sought solutions at the crack tips and at the sharp corners. This has to be achieved by the proper selection of the holomorphic functions used for representation of the complex potentials. The method leads to an overspecified system of linear algebraic equations, which requires regularisation. The latter can be made on the basis of the SVD methods, which also ensures independence of the coefficients in truncated series for complex potentials. Two considered examples indicate that the proposed approach is capable to solve the problems with straight cracks in infinite and finite domains. The analysis of more complex geometries involving curvilinear and branching cracks is required in order to conclude applicability of the suggested approach for the general case.

\section{References}

[1] Muskhelishvili, N.I. Some basic problems of the mathematical theory of elasticity, P. Noordhoff, Groningen, the Netherlands, 1963.

[2] Zielinski, A.P., 1988. Treffz method: elastic and elastoplastic problems. Computer Methods In Applied Mechanics And Engineering, 69, 185-204. 
[3] Kita, E. and Kamiya, N., 1995. Trefftz method: an overview. Advances in Engineering Software, 24, 3-12.

[4] Domingues, J.S., Portela, A. and. Castro, P.M.S.T., 1999. Trefftz boundary element method applied to fracture mechanics. Engineering Fracture Mechanics, 64, 67-86.

[5] Li, Z-C., Lu, T-T. and Hu, H-Y., 2004. The collocation Trefftz method for biharmonic equations with crack singularities. Engineering Analysis with Boundary Elements, 28,79-96.

[6] Galybin, A.N., 2005. Approximation of complex potentials as a uniform approach for solving classical and non-classical boundary value problems of plane elasticity. WIT Transactions on Modelling and Simulation, 39, WIT Press, Southampton, UK, 255-264

[7] Galybin, A.N. and Mukhamediev, Sh.A. 2004. Determination of elastic stresses from discrete data on stress orientations. Int. Journal of Solids and Structures. 41 (18-19), 5125-5142.

[8] Savruk, M.P. Two-dimensional elastic problems for bodies with cracks. Naukova Dumka, Kiev, 1981.

[9] Golub, G.H. and van Loan C.F. Matrix computations, The Johns Hopkins Baltimore / London: University Press, 1989. 